Document downloaded from:

http://hdl.handle.net/10251/77680

This paper must be cited as:

Ferrando Martín, V.; Coves, A.; Andrés, P.; Monsoriu Serra, JA. (2015). Guiding properties of a photonic quasi-crystal fiber based on the thue-morse sequence. IEEE Photonics Technology Letters. 27(18):1903-1906. doi:10.1109/LPT.2015.2444991.

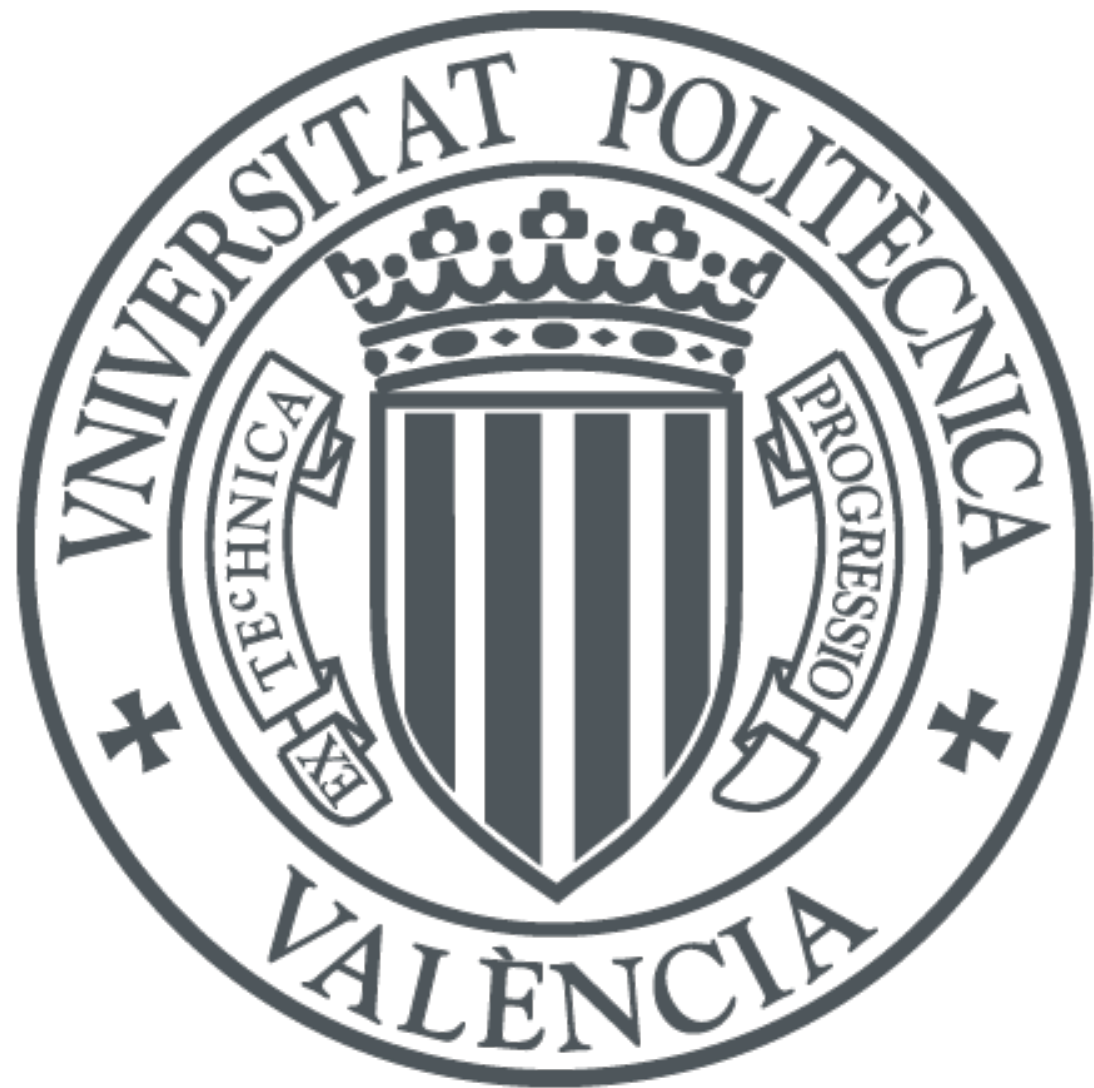

The final publication is available at

http://dx.doi.org/10.1109/LPT.2015.2444991

Copyright Institute of Electrical and Electronics Engineers (IEEE)

Additional Information

(C) 2015 IEEE. Personal use of this material is permitted. Permission from IEEE must be obtained for all other uses, in any current or future media, including reprinting/republishing this material for advertising or promotional purposes, creating new collective works, for resale or redistribution to servers or lists, or reuse of any copyrighted component of this work in other works. 


\title{
Guiding properties of a photonic quasicrystal fiber based on the Thue-Morse sequence
}

\author{
Vicente Ferrando, ${ }^{1,2}$ Ángela Coves, ${ }^{3}$ Pedro Andrés ${ }^{2}$ \\ and Juan A. Monsoriu ${ }^{1, *}$ \\ ${ }^{1}$ Centro de Tecnologías Físicas, Universitat Politècnica de València, E-46022 Valencia, Spain \\ ${ }^{2}$ Departamento de Óptica, Universitat de València, E-46100 Burjassot, Spain \\ ${ }^{3}$ Departamento de Física y Arquitectura de Computadores, Universidad Miguel Hernández, E-03202 Elche, Spain.
}

*jmonsori@fis.upv.es

IEEE Photonics Technology Letters 27, 1903-1906 (2015).

\begin{abstract}
We present a novel microstructured optical fiber having a quasiperiodic distribution of air holes based on the Thue-Morse sequence. The transverse section of these fibers are basically a two-dimensional photonic quasicrystal which can also provide complete photonic band gaps without being a perfect periodic structure. Like in conventional photonic crystal fibers, if the "quasiperiodicity" is broken by decreasing the size of some air holes or by introducing an extra air hole, the modified holes become defects that localize and guide light along the fiber. The guidance is attributed to the inhibition of transverse radiation produced by the photonic quasicrystal cladding. Dispersion curves of guided modes for different structural parameters are calculated, along with the transverse intensity distribution of the fundamental mode. In particular, several specially designed Thue-Morse quasicrystal fibers with nearly-zero ultraflattened group-velocity dispersion are presented.
\end{abstract}

\section{Introduction}

Photonic Crystal Fibers (PCFs) are "in general" thin silica fibers that have a regular array of microscopic holes that extend along the whole fiber length [1]. Any transverse section of the fiber presents an identical 2D silica-air photonic crystal, which for some specific geometries provides a characteristic photonic bandgap structure [2]. If one of these holes is modified, the periodicity is broken and a defect appears in the otherwise regular structure. The well known fact that light can be trapped at defects [3] turns into a propagation feature, being guidance attributed to the inhibition of transverse radiation produced by the 2D photonic crystal cladding [4].

There is a high number of different photonic crystal configurations. The most common PCFs comprises a triangular lattice with a defect generated by the absence of a hole [5]. In this case, donor guided modes appear simultaneously in the upper and the lower forbidden bands of the triangular photonic crystal [6]. Alternatively, guidance 
is also possible in honeycomb fibers in which the defect is generated by an extra hole located off the lattice. An intraband acceptor guided mode appears in the vicinity of the defect [7]. Other configurations have also been proposed in the literature and each of them has its own specific properties. Some examples are the hollow core fibers (PCFs guiding light by an air-core surrounded by 2D lattice) [8] and the bragg fibers (photonic-bandgap fiber formed by concentric rings of multilayer film distributed periodically) [9].

All these configurations with solid-core or air-core have in common claddings characterized by periodic lattices with discrete translational and rotational symmetry that provides a photonic band gaps structure, analogous to the electronic band gaps of a semiconductor. Note that the concept of crystal is usually restricted to periodic order. However, the concept of order without periodicity has been studied extensively in science and technology [10]. In fact, some interesting review articles on photonic quasicrystals can be found in the literature [11,12]. It has been demonstrated that photonic quasicrystals can also exhibit photonic band gaps [13-15]. These aperiodic structures can be generated by deterministic rules and posses long-range order [16]. Unlike periodic structure, aperiodic deterministic structures lack both translational and rotational symmetries but present scale invariance symmetry (self-similarity) in their spectral and structural structure [17]. Fibonacci multilayers is an example of aperiodic system with discrete Fourier spectrum characterized by self-similar bragg peaks which determine the location and width of the frequency band-gap $[18,19]$. Another example of a deterministic aperiodic system is the one-dimensional $[20,21]$ and twodimensional Thue-Morse lattices [22] which also exhibits band gaps phenomena [23]. These photonic quasicrystals can be considered as a square arrangement where some lattice points have been removed following the rules given the aperiodic Thue-Morse sequence in two dimensions.

Within this context, Photonic Quasicrystal Fibers (PQFs) are presented as a new family of microstrutured optical fibers. The transverse section of $\mathrm{PQF}$ consists of a twodimensional Photonic Quasicrystal (the Thue-Morse lattice in our case) of microscopic air holes in silica glass that extend along the entire fiber length (like in conventional PCFs). This kind of fibers may be realized with a standard fabrication process [24] In this work we demonstrate that breaking the "quasiperiodicity" with a defect (with a extra hole or reducing the size of some of them), it is also possible to localize light in the silica core, preventing transverse radiation by the photonic band gap structure provided by the aperiodic lattice. Moreover, the combination of the unusual geometric dispersion behavior shown by the aperiodic lattice, and the material dispersion corresponding to silica core allows us to identify some designs with flattened dispersion profiles at the Ti:sapphire wavelength window and ultraflattened dispersion profiles around $2 \mu \mathrm{m}$.

\section{Design of the Thue-Morse based PQF}

The construction of the one-dimensional Thue-Morse sequence is shown in Fig. 1 up to fourth order. This aperiodic distribution starts at stage $S_{0}$ with the binary value $\{1\}$, where "1" is represented by a blue square in Fig. 1. Then, each element of sequence is obtained iteratively by replacing " 1 " by " 10 " and " 0 " by " 01 ". Therefore, $S_{1}=\{10\}$, $S_{2}=\{1001\}, S_{3}=\{10010110\}$, and so on. In Fig. 1 the elements "0" are represented by red squares.

In a similar way, the two-dimensional Thue-Morse lattice shown in Fig. 2 is con- 


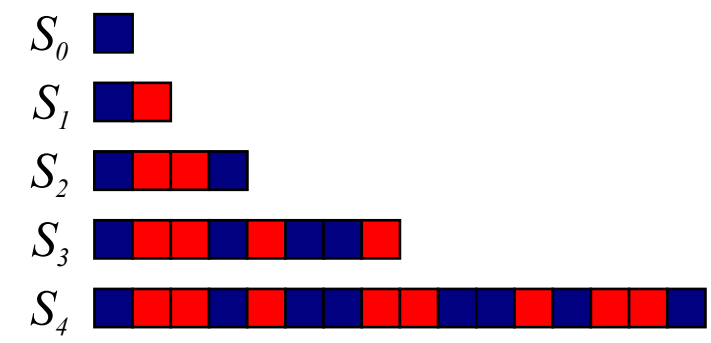

Figure 1: The one-dimensional Thue-Morse sequence up to fourth order.

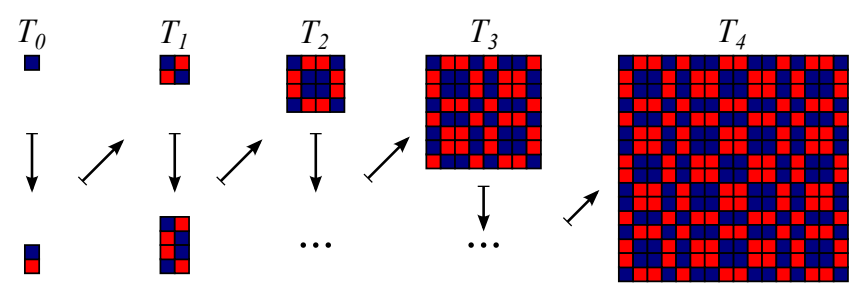

Figure 2: Construction of the two-dimensional Thue-Morse distribution up to fourth order.

structed as follows [25]. Starting from the $1 \times 1$ matrix seed $T_{0}=(0)$, the same transformation rules can be applied, first in the $y$-direction obtaining an intermediate column vector $\left(\begin{array}{l}0 \\ 1\end{array}\right)$. At this point, we apply the Thue-Morse rule to expand each element along the horizontal direction, $x$, obtaining the $2 \times 2$ matrix $T_{1}=\left(\begin{array}{ll}0 & 1 \\ 1 & 0\end{array}\right)$. In order to obtain the next 2D Thue-Morse matrix, we will apply again the $y$ inflation rule on each column of the matrix obtained previously. This step will generate an intermediate $4 \times 2$ matrix. Applying again the $y$-expansion of every element of its rows, we will finally generate a square $4 \times 4$ Thue-Morse matrix, $T_{2}$ (see Fig. 2). Note that the matrix $T_{2}$ can be also obtained from $T_{1}$ using the generalized substitution rules $(0) \rightarrow\left(\begin{array}{ll}0 & 1 \\ 1 & 0\end{array}\right)$ and $(1) \rightarrow\left(\begin{array}{ll}1 & 0 \\ 0 & 1\end{array}\right)$. Higher-order Thue-Morse matrices, $T_{n}$, can be simply obtained by repeating the same iteration scheme up to desired generation order. Mathematically, it is also possible to obtain each element of the 2D Thue-Morse matrix of order $n$ as $\left[T_{n}\right]_{i, j}=\left[S_{n}\right]_{i} \odot\left[S_{n}\right]_{j}$, where $\left[S_{n}\right]_{i}$ and $\left[S_{n}\right]_{i}$ are is the $i$ th and $j$ th element, respectively, of the 1D Thue-Morse vector $S_{n}$, and $\odot$ represents the XNOR function.

From this geometrical representation two designs of PQFs based on the ThueMorse lattice of order four can be constructed (see Fig. 3). The first microstructured optical fibers corresponds to the air holes being located at the center of the blue squares (direct Thue-Morse fiber) of Fig. 2, the second in the center of the red squares (inverse Thue-Morse fiber). Note that the cladding of these PQFs can be considered as a rectangular arrangement of air holes in silica [26] where some of them have been removed according to the Thue-Morse rules. The core of the fibers is performed by reducing the size of the central holes in the direct fiber or by introducing an extra central hole in the inverse fiber. In this way, donor and acceptor defects are considered in the direct and inverse fibers, respectively, like in conventional PCF [4].

The concepts of donor and acceptor modes comes from semiconductor Physics. The addition of donor impurities contributes electron energy levels high in the semiconductor band gap. On the other hand, the addition of acceptor impurities contributes hole levels low in the semiconductor band gap. Within the context of microstructured 

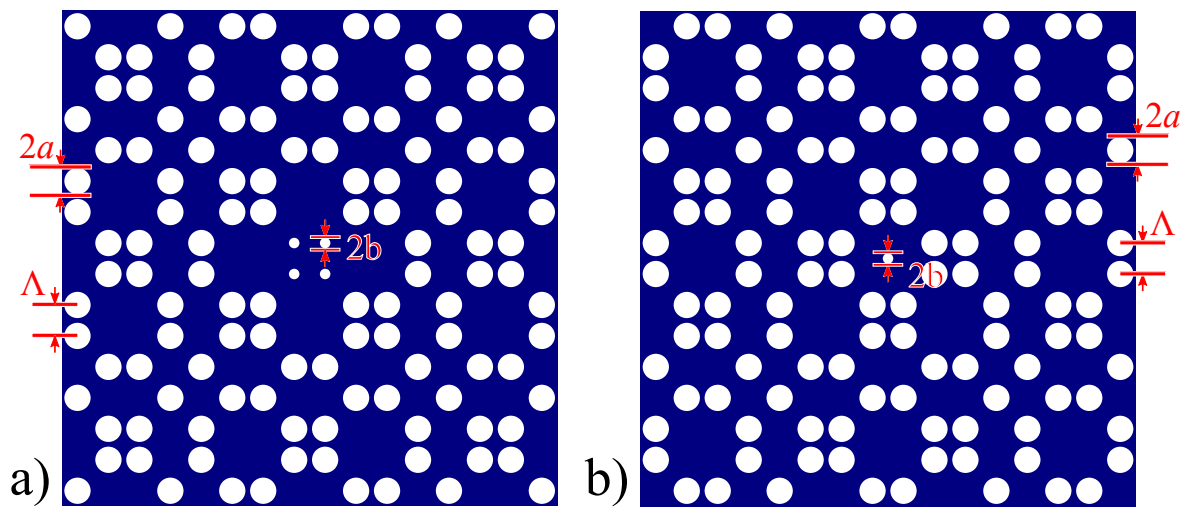

Figure 3: a) Transverse section of two models of PQFs based on the 2D Thue-Morse lattice of order four: a) Direct Thue-Morse fiber where the air holes are located at the center of the blue squares in Fig. 2 and a donor defect is introduced by reducing the size of the central holes. b) Inverse Thue-Morse fiber where the air holes are located at the center of the red squares in Fig. 2 and an acceptor defect is introduced as a central extra hole.

fibers, a donor defect performed by reducing the size of the central air-holes generates guided modes into the forbidden bands from the neighboring conduction bands. In a similar way, an acceptor defect in the 2D lattice (an extra air-hole) causes guided modes to leave the upper conduction band and to enter the forbidden band located just below [4]. In this work we demonstrate that PQFs are governened by the same guiding mechanism when the quasiperiodicity is broken with a defect.

\section{Guiding and dispersion properties}

We have simulated different direct and inverse PQFs based on the Thue-Morse sequence. The structural parameter that define PQF geometry are the "quasiperiodicity", $\Lambda$, the radius of the air holes, $a$, and the radius of the central air holes, $b$, that define the donor or acceptor defects in the fiber, as depicted in Fig. 3. Our numerical calculations are performed with a 2D full-vector modal method that incorporates the material dispersion in a natural way [27]. Figure 4 shows the two different band-gap structures and the modal dispersion curves for the guided modes in silica-air direct and inverse PQFs. Reducing the size of the four central air holes in the direct fiber $(b<a)$, guided modes appear simultaneously above and below the first conduction band. These donor guided modes arise from the next lower conduction band when the ratio $b / a$ is reduced, moving through the forbidden band provided by the aperiodic lattice. In particular, the guided mode shown in the upper semi-infinite forbidden band in Fig. 4(a) is the fundamental mode of the direct PQF. On the other hand, Figure 4(b) shows how introducing an extra air hole in the center of the inverse Thue-Morse lattice, an acceptor guided mode leaves the upper conduction band and enters the forbidden band located just below, so the fundamental guided mode for this configuration is intraband. Therefore, it is demonstrated that intraband guidance phenomena are also possible with aperiodic lattices as in conventional PCF [4]. The transverse irradiance distribution for the fundamental guided modes in direct and inverse PQFs are shown in Fig. 5(a) and 


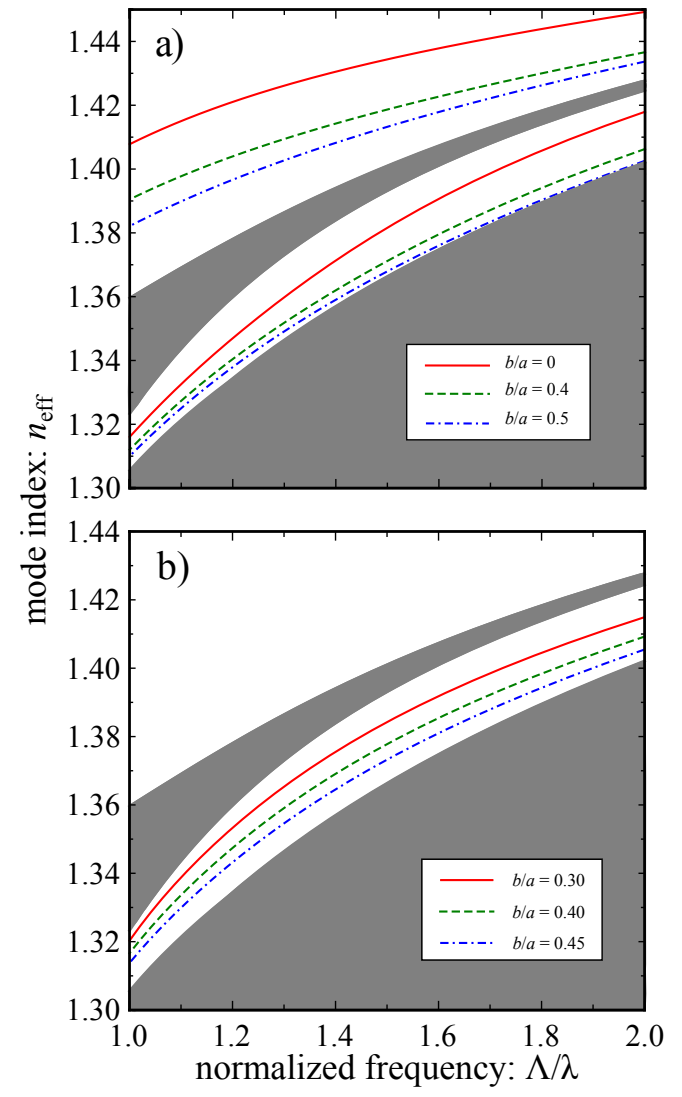

Figure 4: a) Modal dispersion curve for three donor modes characterized by different ratios $b / a$ of the four central holes in the direct PQF with a inverse normalized radium $\Lambda / a=2.357$. b) Modal dispersion curve for three acceptor modes characterized by different ratios $b / a$ of the central extra hole in the inverse PQF with a inverse normalized radium $\Lambda / a=2.357$. Shaded areas correspond to the conduction bands formed by the structure radiation modes, while the white areas are the forbidden bands.

5(b), respectively, for a wavelength $\lambda=800 \mathrm{~nm}$. Calculations reveal that the fundamental modes in direct and inverse PQFs are, in fact, linear polarization doublets, as a consequence of the discrete rotational symmetry of the fibers.

In order to analyze the dispersion properties of PQFs based in the Thue-Morse sequence, we have simulated a number of different direct and inverse designs by changing the equivalent quasiperiod, $\Lambda$, the hole radius of the aperiodic lattice, $a$, and the hole radius of the defects, $b$. In this way, by analyzing the dispersion curves of these different PQFs, we have found that it is possible to control their dispersion characteristics following a procedure similar to that described in [28]. The group velocity dispersion, $D$, including the material dispersion, can be evaluated as

$$
D(\lambda)=-\frac{\lambda}{c} \frac{d^{2} n_{\mathrm{eff}}}{d \lambda^{2}}
$$

where $n_{\mathrm{eff}}$ is the effective refractive index of the guided mode. As a first approximation, 
a)

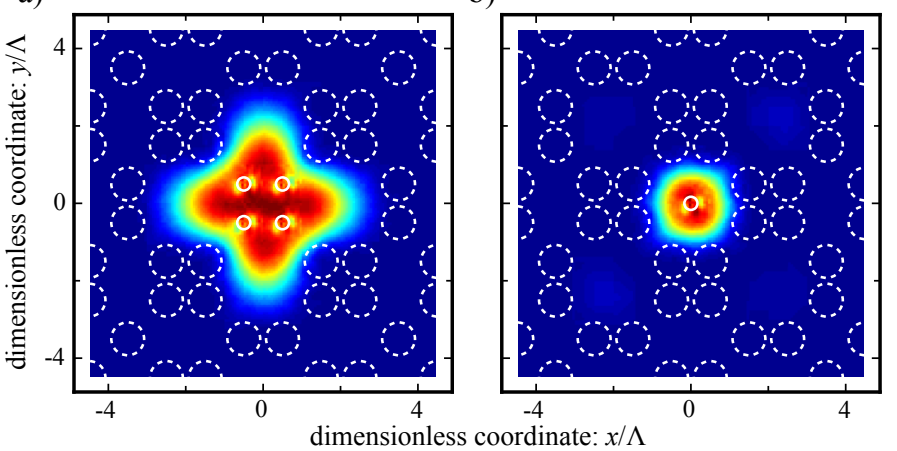

Figure 5: Transverse irradiance for the fundamental guided mode of the a) direct and b) inverse PQF described in Fig. 3. In both cases, $\Lambda=424 \mathrm{~nm}, a=180 \mathrm{~nm}, b / a=0.4$, and $\lambda=800 \mathrm{~nm}$.

the dispersion of a magnified structure, $D_{M}$, is given by [29]

$$
D_{M}(\lambda) \approx \underbrace{\frac{1}{M}\left\{D\left(\frac{\lambda}{M}\right)-D_{m}\left(\frac{\lambda}{M}\right)\right\}}_{D_{g, M}(\lambda)}+D_{m}(\lambda),
$$

being $M=\Lambda / \Lambda_{0}$ the magnification with respect to a reference structure with $\Lambda=\Lambda_{0}$ the dispersion of which is $D$, and $D_{m}$ the material dispersion of the reference material (silica in this case). The first term in the right-hand side of Eq. (2) corresponds to $D_{g, M}$, the geometric dispersion of the magnified structure, i.e., the dispersion of the magnified fiber with the condition that the material is non-dispersive. The above equations allows us to obtain the structural parameters that provides approximately the expected dispersion behavior. Then, we can tune these parameters with the exact Eq. (1) to obtain the desired dispersion profile. Using this procedure, it is possible to obtain interesting dispersion configurations with PQFs based on the Thue-Morse lattice. In order to reduce the design parameters, we have considered the direct PQF shown in Fig. 3(a) with a solid core $(b=0)$. The fundamental guided mode for this configuration looks like Fig. 5(a) without the effect shown around the small central air holes. In Fig. 6(a) we present some example of flattened designs with a zero third-ordered dispersion point centered at different wavelengths. It is clear the tunability of the structure. We can appreciate how the flattened dispersion curves are displaced along the wavelength axis and are broaden as the ratio $\Lambda / a$ increases. It is remarkable the possibility of obtaining a constant dispersion at Ti:sapphire wavelength window around $0.8 \mu \mathrm{m}$ (red curve). Further increasing the ratio $\Lambda / a$, we have obtained in Fig. 6(b) three different ultraflattened dispersion designs in the long wavelength communication window around $2 \mu \mathrm{m}$ improving in this way the performance of standard microstructured fibers with ultraflatten behavior around $1.55 \mu \mathrm{m}[9,30]$. These dispersion curves present two points with zero third-ordered dispersion and a middle point with zero fourth-ordered dispersion. The tunability of this aperiodic fiber is also clearly demonstrated by the fact that these ultraflattened designs own positive, nearly zero, and negative $D$. 


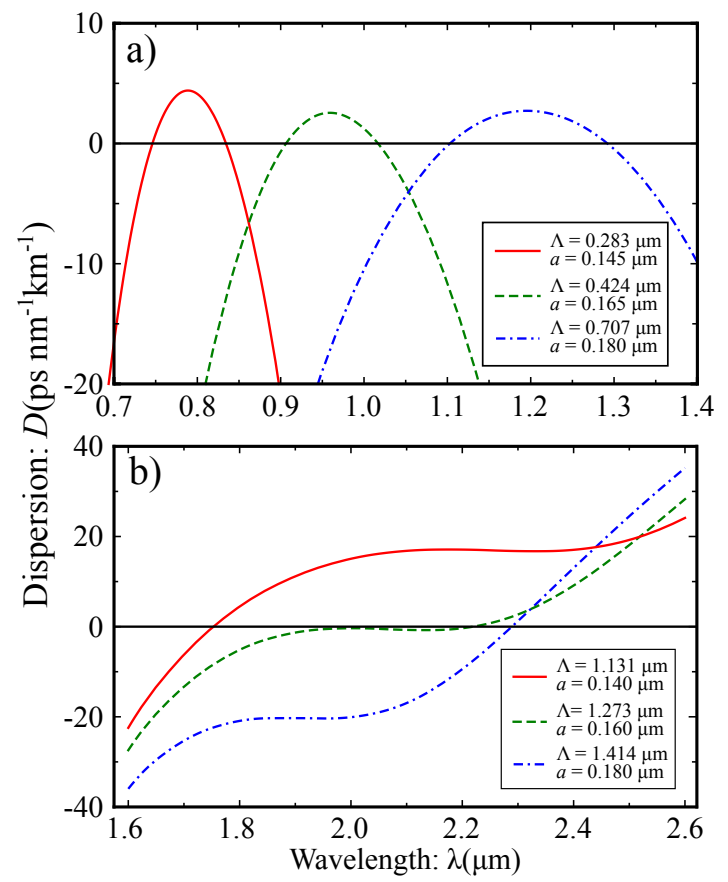

Figure 6: Dispersion versus wavelength for the fundamental guided mode of the direct Thue-Morse structure shown in Fig. 3(a) with a solid core $(b=0)$. a) Flattened dispersion curves. b) Ultraflattened dispersion curves.

\section{Conclusions}

In this work we have designed a new type of microstructured optical fiber based on aperiodic series. In particular, a two-dimensional structure based on the Thue-Morse sequence is presented. After demonstrating that the designed structure is able to guide modes, as conventional PCFs, by introducing both donor and acceptor defects in the quasiperiodic structure, we have studied the dispersion behavior under the manipulation of the design parameters, reaching some interesting configurations with flatten group-velocity dispersion around $800 \mathrm{~nm}$ and a tunable ultraflatten group-velocity dispersion at the $2 \mu \mathrm{m}$ band.

\section{Acknowledgements}

The authors thank Dr. Enrique Silvestre for his useful comments and suggestions. We also acknowledge the financial support from Ministerio de Economía y Competitividad (grants TEC2013-46643-C2-1-R and FIS2011-23175) and the Generalitat Valenciana (grants PROMETEOII/2014/072 and ACOMP/2014/180), Spain.

\section{References}

[1] J. C. Knight, "Photonic Band Gap Guidance in Optical Fibers," Science 282, 1476-1478 (1998). 
[2] E. Yablonovitch, "Photonic band-gap structures," J. Opt. Soc. Am. B 10, 283-295 (1993).

[3] E. Yablonovitch, T. Gmitter, R. Meade, A. Rappe, K. Brommer, and J. Joannopoulos, "Donor and acceptor modes in photonic band structure," Phys. Rev. Lett. 67, 3380-3383 (1991).

[4] A. Ferrando, E. Silvestre, J. J. Miret, P. Andrés, and M. V. Andrés, "Donor and acceptor guided modes in photonic crystal fibers," Opt. Lett. 25, 1328-1330 (2000).

[5] P. S. Russell, "Photonic-Crystal Fibers," J. Lightwave Technol. 24, 4729-4749 (2006).

[6] A. Ferrando, E. Silvestre, J. J. Miret, and P. Andrés, "Vector description of higherorder modes in photonic crystal fibers," J. Opt. Soc. Am. A 17, 1333-1340 (2000).

[7] S. E. Barkou, J. Broeng, and A. Bjarklev, "Silica-air photonic crystal fiber design that permits waveguiding by a true photonic bandgap effect," Opt. Lett. 24, 46-48 (1999).

[8] P. J. Roberts, F. Couny, H. Sabert, B. J. Mangan, D. P. Williams, L. Farr, M. W. Mason, A. Tomlinson, T. A. Birks, J. C. Knight, and P. S. Russell, "Ultimate low loss of hollow-core photonic crystal fibres," Opt. Express 13, 236-244 (2005).

[9] J. A. Monsoriu, E. Silvestre, A. Ferrando, P. Andrés, and J. J. Miret, "High-indexcore Bragg fibers: dispersion properties," Opt. Express 11, 1400-1405 (2003).

[10] E. Maciá, "The role of aperiodic order in science and technology," Rep. Prog. Phys. 69, 397-441 (2006).

[11] W. Steurer and D. Sutter-Widmer, "Photonic and phononic quasicrystals," J. Phys. D: Appl. Phys. 40, R229-R247 (2007).

[12] Z. V. Vardeny, A. Nahata, and A. Agrawal, "Optics of photonic quasicrystals," Nature Photon. 7, 177-187 (2013).

[13] M. E. Zoorob, M. D. Charlton, G. J. Parker, J. J. Baumberg, and M. C. Netti, "Complete photonic bandgaps in 12-fold symmetric quasicrystals," Nature 404, 740-743 (2000)

[14] Y. S. Chan, C. T. Chan, and Z. Y. Liu, "Photonic Band Gaps in Two Dimensional Photonic Quasicrystals," Phys. Rev. Lett. 80, 956-959 (1998).

[15] C. Jin, B. Cheng, B. Man, Z. Li, D. Zhang, S. Ban, and B. Sun, "Band gap and wave guiding effect in a quasiperiodic photonic crystal," Appl. Phys. Lett. 75, 1848 (1999).

[16] E. Maciá, "Exploiting aperiodic designs in nanophotonic devices," Rep. Prog. Phys. 75, 036502 (2012).

[17] P. V. Korolenko, A. Y. Mishin, and Y. V. Ryzhikova, "Scaling in the characteristics of aperiodic multilayer structures," J. Opt. Technol. 79, 754-757 (2012).

[18] W. J. Hsueh, C. H. Chang, Y. H. Cheng, and S. J. Wun, "Effective Bragg conditions in a one-dimensional quasicrystal," Opt. Express 20, 26618-26623 (2012). 
[19] G. Gumbs, G. S. Dubey, A. Salman, B. S. Mahmoud, and D. Huang, "Statistical and transport properties of quasiperiodic layered structures: Thue-Morse and Fibonacci," Phys. Rev. B 52, 210-219 (1995).

[20] H. Alipour-Banaei, F. Mehdizadeh, and M. Hassangholizadeh-Kashtiban, "Important effect of defect parameters on the characteristics of Thue-Morse photonic crystal filters," Adv. Optoelectron. 2013, 856148 (2013).

[21] X. Jiang, Y. Zhang, S. Feng, K. C. Huang, Y. Yi, and J. D. Joannopoulos, "Photonic band gaps and localization in the ThueâMorse structures," Appl. Phys. Lett. 86, 201110 (2005).

[22] L. Moretti and V. Mocella, "Two-dimensional photonic aperiodic crystals based on Thue-Morse sequence," Opt. Express 15, 15314-15323 (2007).

[23] S. V. Boriskina, A. Gopinath, and L. Dal Negro, "Optical gap formation and localization properties of optical modes in deterministic aperiodic photonic structures," Opt. Express 16, 18813-18826 (2008).

[24] P. Russell, E. Marin, A. Diez, S. Guenneau, and A. Movchan, "Sonic band gaps in PCF preforms: enhancing the interaction of sound and light," Opt. Express 11, 2555 (2003).

[25] L. D. Negro, N.-N. Feng, and A. Gopinath, "Electromagnetic coupling and plasmon localization in deterministic aperiodic arrays," J. Opt. A: Pure Appl. Opt. 10, 064013 (2008).

[26] A. Bouk, A. Cucinotta, F. Poli, and S. Selleri, "Dispersion properties of squarelattice photonic crystal fibers," Opt. Express 12, 941-946 (2004).

[27] E. Silvestre, T. Pinheiro-Ortega, P. Andrés, J. J. Miret, and A. Ortigosa-Blanch, "Analytical evaluation of chromatic dispersion in photonic crystal fibers," Opt. Letters 30, 453-455 (2005).

[28] A. Ferrando, E. Silvestre, P. Andrés, J. J. Miret, and M. V. Andrés, "Designing the properties of dispersion-flattened photonic crystal fibers," Opt. Express 9, 687697 (2001)

[29] E. Silvestre, T. Pinheiro-Ortega, P. Andrés, J. J. Miret, and A. Coves, "Differential toolbox to shape dispersion behavior in photonic crystal fibers," Opt. Lett. 31, 1190-1192 (2006).

[30] A. Ferrando, E. Silvestre, J. J. Miret, and P. Andrés, "Nearly zero ultraflattened dispersion in photonic crystal fibers," Opt. Lett. 25, 790-792 (2000). 